\title{
Diabetes and its Effects: Statistics and Biosensors
}

\section{Kanchi $\mathbf{S}^{1^{*}}$, Sharma $\mathbf{D}^{1}$, Bisetty $\mathbf{K}^{1^{*}}$, Nuthalapati VN ${ }^{2}$}

${ }^{1}$ Department of Chemistry, Durban University of Technology, P.O Box 1334, Durban 4000, South Africa

${ }^{2}$ Department of Chemistry, Sri Venkateswara University, Tirupati-517 502, A.P., India

\section{Introduction}

The term "diabetes mellitus" describes a metabolic disorder of multiple aetiology characterized by chronic hyperglycaemia with disturbances of carbohydrate, fat and protein metabolism resulting from defects in insulin secretion, insulin action, or both. The effects of diabetes mellitus include long-term damage, dysfunction and failure of various organs. Diabetes is categorized into Type- 1 and Type-2. Type1 diabetes was previously called insulin dependent diabetes mellitus or juvenile-onset diabetes. Although disease onset can occur at any age, the peak age for diagnosis is in the mid-teens. Type 1 diabetes develops when the cells that produce the hormone insulin, known as the beta cells, in the pancreas are destroyed. This destruction is initiated or mediated by the body's immune system and limits or completely eliminates the production and secretion of insulin, the hormone that is required to lower blood glucose levels. To survive, people with Type-1 diabetes must have insulin delivered by injection or a pump. In adults, Type- 1 diabetes accounts for approximately $5 \%$ of all diagnosed cases of diabetes.

Type-2 diabetes was previously called non-insulin dependent diabetes mellitus or adult-onset diabetes because the peak age of onset is usually later than Type- 1 diabetes. In adults, Type- 2 diabetes accounts for about 90 to $95 \%$ of all diagnosed cases of diabetes. Type2 diabetes usually begins with insulin resistance, a disorder in which the cells primarily within the muscles, liver, and fat tissue do not use insulin properly. As the need for insulin rises, the beta cells in the pancreas gradually lose the ability to produce sufficient quantities of the hormone. The role of insulin resistance as opposed to beta cell dysfunction differs among individuals, with some having primarily insulin resistance and only a minor defect in insulin secretion, and others with slight insulin resistance and primarily a lack of insulin secretion. The risk for developing Type-2 diabetes is associated with older age, obesity, family history of diabetes, history of gestational diabetes, impaired glucose metabolism, physical inactivity, and race/ ethnicity. African Americans, Hispanics/Latinos, American Indians, some Asians, and Native Hawaiians or other Pacific Islanders are at particularly high risk for Type-2 diabetes and its complications. Type- 2 diabetes in children and adolescents, although uncommon, is being diagnosed more frequently among American Indians, African Americans, Hispanics/Latinos, Asians, and Pacific Islanders.

Pre-diabetes is a condition in which individuals have high blood glucose or hemoglobin A1C levels but not high enough to be classified as diabetes. People with pre-diabetes have an increased risk of developing Type- 2 diabetes, heart disease, and stroke, but not everyone with pre-diabetes will progress to diabetes. The Diabetes Prevention Program, a large prevention study of people at high risk for diabetes, showed that lifestyle intervention that resulted in weight loss and increased physical activity in this population can prevent or delay Type-2 diabetes and in some cases return blood glucose levels to within the normal range. Other international studies have shown similar results [1]. Other categories of diabetes include gestational diabetes (a state of hyperglycemia which develops during pregnancy) and "other" rarer causes (genetic syndromes, acquired processes such as pancreatitis, diseases such as cystic fibrosis, and exposure to certain drugs, viruses, and unknown causes). As well, intermediate states of hyperglycemia (impaired fasting glucose or impaired glucose tolerance) have been defined. These states are significant in that they can progress to diabetes, but with weight loss and lifestyle changes, this progression can be prevented or delayed. In the short term, hyperglycemia causes symptoms of increased thirst, increased urination, increased hunger, and weight loss. However, in the long-term, it causes damage to eyes (leading to blindness), kidneys (leading to renal failure), and nerves (leading to impotence and foot disorders/possibly amputation). As well, it increases the risk of heart disease, stroke, and insufficiency in blood flow to legs. Studies have shown that good metabolic control prevents or delays these complications. Thus, the primary goal of treatment is to bring the elevated blood sugars down to a normal range, both to improve symptoms of diabetes as well as to prevent or delay diabetic complications. Achieving this goal requires a comprehensive, coordinated, patient-centred approach on the part of the health care system.

According to the statistic obtained from the literature, 387 million people have diabetes and it can be increased to 592 million by the year 2035. In every country the number of people with Type- 2 diabetes is increasing day-by-day. The $77 \%$ of pollution with diabetes live in low- and middle-income countries. The greatest number of people with diabetes is between 40 and 59 years of age. The surprising data found form the literature that 179 million people with diabetes are undiagnosed and also for every seven seconds a person dies from diabetes. The total deaths in 2014 with diabetes were 4.9 million. In $2014,11 \%$ of 612 billion dollars of US health expenditure was invested on adult diabetic people. In 2013, more than 79,000 children were developed Type -1diabetes worldwide. More than 21 million live births were affected by diabetes during pregnancy in 2013. In Africa, $76 \%$ of deaths due to diabetes are in people under the age of 60 and in the Middle East and North Africa, 1 in 10 adults has diabetes. Europe has the highest prevalence of type 1 diabetes in children. The governments of North America and Caribbean are spending more on healthcare for diabetes in North America than countries. In South and Central America, the number of people with diabetes will increase by $60 \%$ by 2035 . In South-East Asia, almost half of people with diabetes are undiagnosed. In the Western Pacific, 138 million adults have diabetes the largest number of any region [2] as shown in Figure 1.

Diabetes remained the $7^{\text {th }}$ leading cause of death in the United States in 2010, with 69,071 death certificates listing it as the underlying cause of death, and a total of 234,051 death certificates listing diabetes as an underlying or contributing cause of death. As per to the 2014 National Diabetes Statistics Report, in 2012, 29.1 million Americans had diabetes and approximately 1.25 million American children and

*Corresponding author: Kanchi S, Bisetty K, Department of Chemistry, Durban University of Technology, South Africa, Tel: +27-373-6008/2311; Email: ksuvardhan@gmail.com, bisettyk@dut.ac.za

Received April 27, 2014; Accepted April 28, 2015; Published April 30, 2015

Citation: Kanchi S, Sharma D, Bisetty K, Nuthalapati VN (2015) Diabetes and its Effects: Statistics and Biosensors. J Environ Anal Chem 2: e111. doi:10.4172/2380-2391.1000e111

Copyright: @ 2015 Kanchi S, et al. This is an open-access article distributed under the terms of the Creative Commons Attribution License, which permits unrestricted use, distribution, and reproduction in any medium, provided the original author and source are credited. 
Citation: Kanchi S, Sharma D, Bisetty K, Nuthalapati VN (2015) Diabetes and its Effects: Statistics and Biosensors. J Environ Anal Chem 2: e111. doi:10.4172/2380-2391.1000e111

Page 2 of 4

adults have Type-1 diabetes. Of the 29.1 million, 21.0 million were diagnosed, and 8.1 million were undiagnosed. The percentage of Americans age 65 and older remains high, at $25.9 \%$, or 11.8 million seniors (diagnosed and undiagnosed). The incidence of diabetes in 2012 was 1.7 million new diagnoses/year; in 2010 it was 1.9 million. In 2012, 86 million Americans age 20 and older had pre-diabetes; this is up from 79 million in 2010. About 208,000 Americans under age 20 are estimated to have diagnosed diabetes, approximately $0.25 \%$ of that population. In 2008-09, the annual incidence of diagnosed diabetes in youth was estimated at 18,436 with Type- 1 diabetes, 5,089 with Type-2 diabetes. The statistics of effected diabetic patients in United States of America [3] was shown in Figure 2.

\section{Biosensors for Glucose Detection}

A biosensor is an analytical device, used for the detection of an analyte that combines a biological component with a physicochemical detector. The sensitive biological element (e.g. tissue, microorganisms, organelles, cell receptors, enzymes, antibodies, nucleic acids, etc.), a biologically derived material or biomimetic component that interacts (binds or recognizes) the analyte under study. The biologically sensitive elements can also be created by biological engineering. The transducer or the detector element (works in a physicochemical way; optical, piezoelectric, electrochemical, etc.) that transforms the signal resulting from the interaction of the analyte with the biological
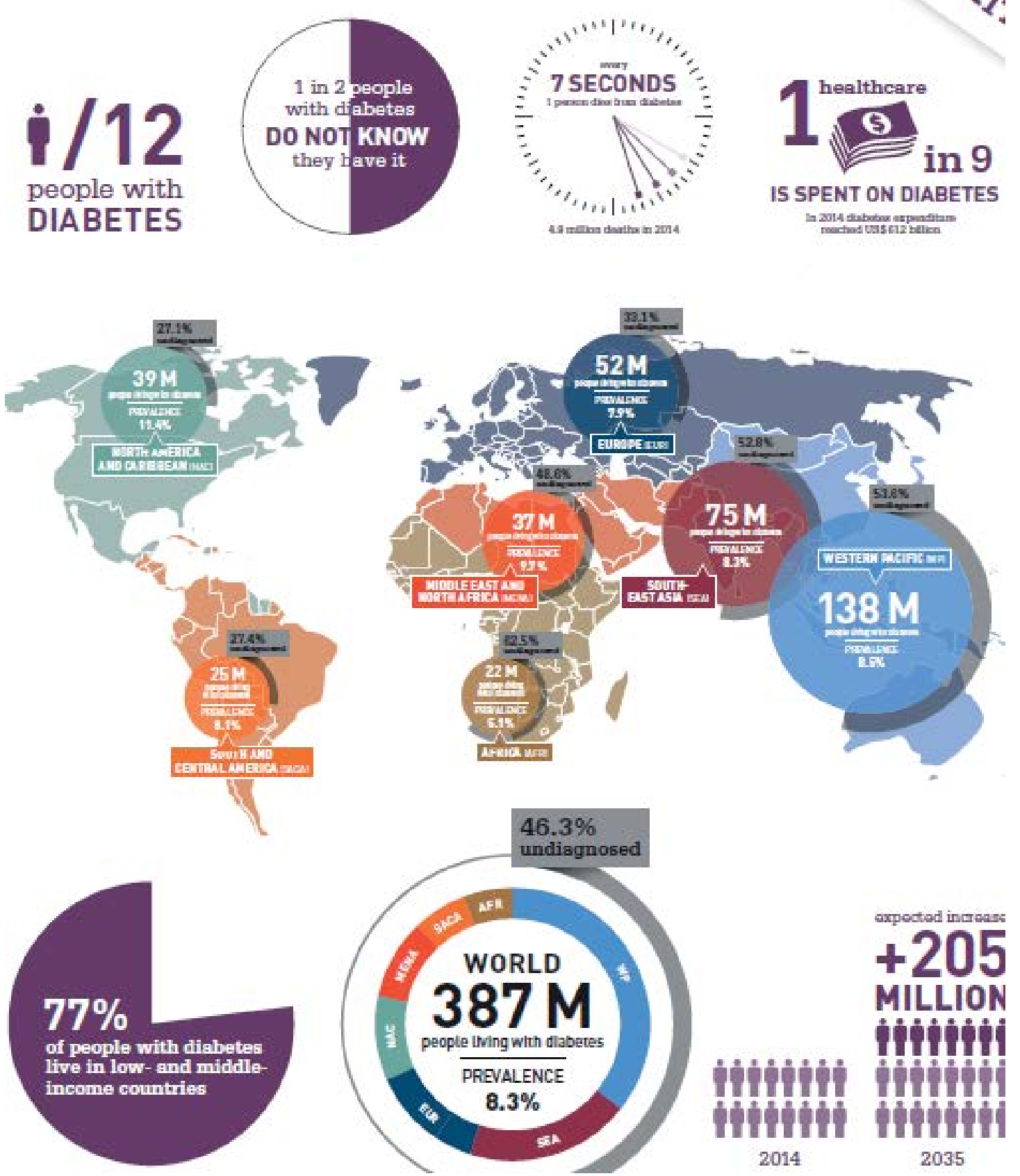

Figure 1: World's diabetic statistics [2]. 
Citation: Kanchi S, Sharma D, Bisetty K, Nuthalapati VN (2015) Diabetes and its Effects: Statistics and Biosensors. J Environ Anal Chem 2: e111. doi:10.4172/2380-2391.1000e111

\section{Percentage(\%)}

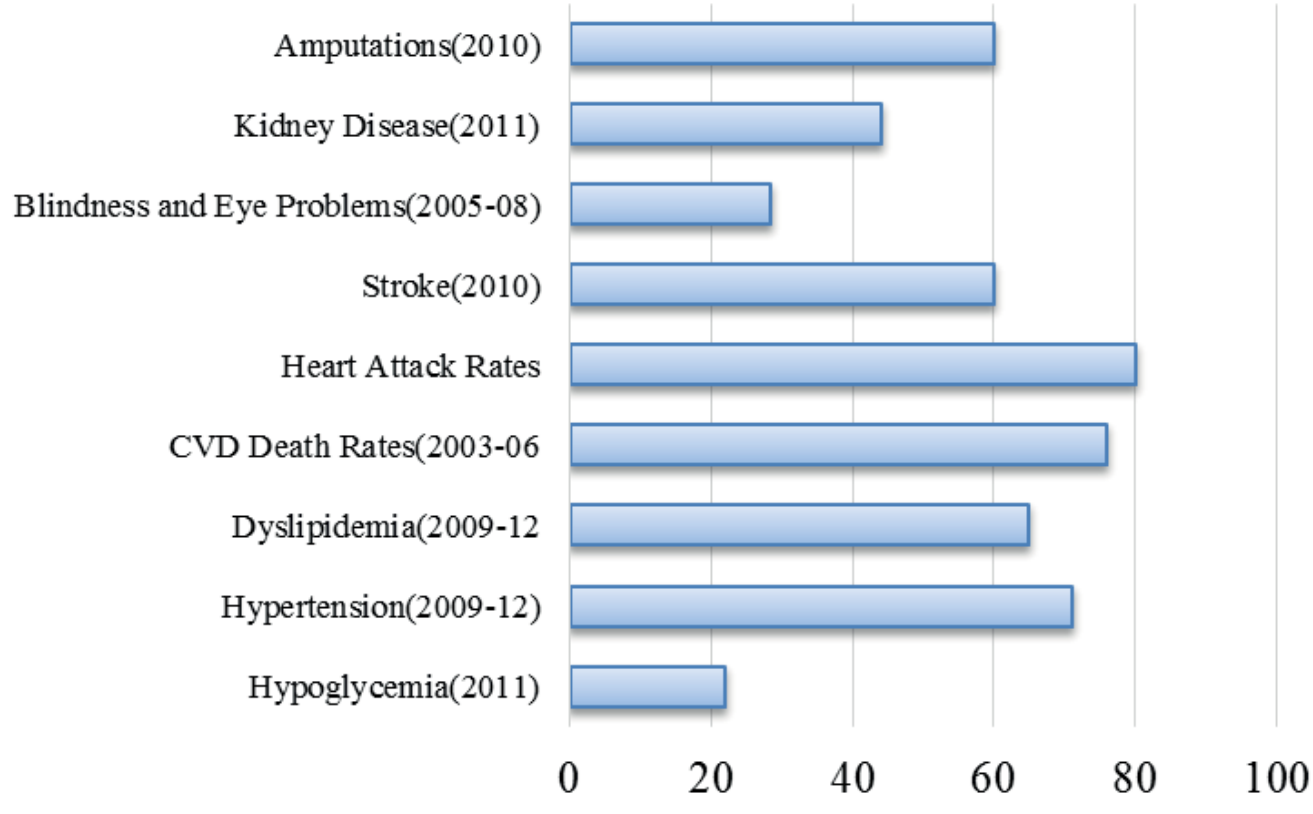

Figure 2: The statistics of diabetic patients in United States of America from 2005-2012 with different health issues [3].

element into another signal (i.e., transduces) that can be more easily measured and quantified. The biosensor reader device with the associated electronics or signal processors that are primarily responsible for the display of the results in a user-friendly way. This sometimes accounts for the most expensive part of the sensor device; however it is possible to generate a user friendly display that includes transducer and sensitive element. Amperometric enzyme electrodes, based on glucose oxidase (GOx), have played a leading role in the move to simple easyto-use blood sugar testing and are expected to play a similar role in the move toward continuous glucose monitoring. Since Clark and Lyons proposed in 1962 the initial concept of glucose enzyme electrodes [4,5], we have witnessed tremendous effort directed toward the development of reliable devices for diabetes control. Different approaches have been explored in the operation of glucose enzyme electrodes. In addition to diabetes control, such devices offer great promise for other important applications, ranging from bioprocess monitoring to food analysis.

\section{Evolution of glucose biosensors}

In 1962 the first glucose monitoring device was introduced by Clark and Lyons [3]. The first glucose enzyme electrode relied on a thin layer of GOx entrapped over an oxygen electrode via a semipermeable dialysis membrane. Measurements were made based on the monitoring of the oxygen consumed by the enzyme-catalyzed reaction and negative potential was applied to the platinum cathode for a reductive detection of the oxygen consumption

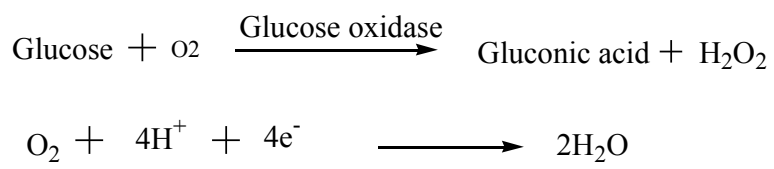

First-generation glucose biosensors rely on the use of the natural oxygen cosubstrate and generation and detection of hydrogen peroxide. Later stages, biosensor depends on the biocatalytic reaction which involves reduction of the flavin group (FAD) in the enzyme by reaction with glucose to give the reduced form of the enzyme $\left(\mathrm{FADH}_{2}\right)$ followed by re-oxidation of the flavin by molecular oxygen to regenerate the oxidized form of the enzyme GOx (FAD).

$$
\begin{array}{ll}
\mathrm{GOx}(\mathrm{FAD})+\text { Glucose } & \mathrm{GOx}\left(\mathrm{FADH}_{2}\right)+\text { Gluconolactone } \\
\mathrm{GOx}\left(\mathrm{FADH}_{2}\right)+\mathrm{O}_{2} \longrightarrow \mathrm{GOx}(\mathrm{FAD})+\mathrm{H}_{2} \mathrm{O}_{2}
\end{array}
$$

The measurements of peroxide formation have the advantage of being simpler, especially when miniaturized devices are concerned.

In later stages, biosensors are further improved by replacing the oxygen with a non-physiological (artificial) electron acceptor capable of shuttling electrons from the redox center of the enzyme to the surface of the electrode.

The transfer of electrons between the GOx active site and the electrode surface is the limiting factor in the operation of amperometric glucose biosensors. Glucose oxidase does not directly transfer electrons to conventional electrodes because of a thick protein layer surrounding its flavin adenine dinucleotide (FAD) redox center and introducing an intrinsic barrier to direct electron transfer.

$$
\begin{gathered}
\text { Glucose }+\mathrm{GOx}_{\text {(oxidation) }} \longrightarrow \text { Gluconic acid }+\mathrm{GOx}_{\text {(reduction) }} \\
\text { GOx(reduction) }+2 \mathrm{M}_{\text {(oxidation) }} \longrightarrow \mathrm{GOx}_{\text {(oxidation) }}+2 \mathrm{M}_{\text {(reduction) }}+2 \mathrm{H}^{+} \\
2 \mathrm{M}_{\text {(reduction) }} \longrightarrow 2 \mathrm{M}_{\text {(oxidation) }}+2 \mathrm{e}^{-}
\end{gathered}
$$

Chemical modification of GOx with electron-relay groups represents another attractive route for facilitating the electron transfer between the GOx redox center and the electrode surface. The emergence 
of nanotechnology has opened new horizons for the application of nanomaterials in bioanalytical chemistry. Recent advances in nanotechnology offer exciting prospects in the field of bioelectronics. Owing to the similar dimensions of nanoparticles and redox proteins such nanomaterials can be used for effective electrical wiring of redox enzymes. Various nanomaterials, including gold nanoparticles or carbon nanotubes (CNT), have thus been used as electrical connectors between the electrode and the redox center of GOx.

Low-cost biosensors are in high demands in many areas including cancer and diabetes diagnosis. Among the various types of biosensors, electrochemical biosensors exhibit great advantages such as high sensitivity, rapid response, simplicity, compatibility with miniaturization technology, and low cost. Advancement in the technology leads to the development of a reagent-less glucose biosensor with a low operating potential, close to that of the redox potential of the enzyme. In this case, the electron is transferred directly from glucose to the electrode via the active site of the enzyme. The absence of mediators is the main advantage of such third-generation biosensors, leading to a very high selectivity.

\section{Challenges}

Despite the impressive progress in the development of glucose biosensors, the promise of tight diabetes management has not been fulfilled, and there are still many challenges and obstacles related to the achievement of a highly stable and reliable continuous glycemic monitoring. Such monitoring of moment-to-moment changes in blood glucose concentrations is expected to lead to a substantial improvement in the management of diabetes. The motivation of providing such tight diabetes control thus remains the primary focus of many researchers. Clearly, success in this direction demands a detailed understanding of the underlying biochemistry, physiology, surface chemistry, electrochemistry, and material chemistry. Yet, the ultimate implementation of the new devices may rely on commercial and legal considerations rather than scientific ones.

\section{References}

1. Centers for Disease Control and Prevention (2014) National Diabetes Statistics Report: Estimates of Diabetes and Its Burden in the United States. Department of Health and Human Services, Atlanta, GA USA.

2. International Diabetes Federation (2014) IDF Diabetes Atlas (6thedn.)

3. National Diabetes Statistics Report (2014).

4. Clark LC, Lyons C (1962) Electrode systems for continuous monitoring in cardiovascular surgery. Ann N Y Acad Sci 102: 29-45.

5. Wang J (2008) Electrochemical Glucose Biosensors. Chem Rev 108: 814-825. 